\title{
Obesity in Polycystic Ovary Syndrome: Insulin Sensitizing Therapy
}

\author{
Kathleen M. Hoeger
}

Published online: 19 August 2012

(C) Springer Science+Business Media, LLC 2012

\begin{abstract}
Polycystic Ovary Syndrome (PCOS) is a common reproductive endocrine disorder in women that is highly associated with obesity. Whether obesity is intrinsic to the disorder or is a result of different lifestyle and environmental concerns is unclear, however obesity influences the risks of PCOS with respect to fertility complications, pregnancy complications and cardiovascular risk. Polycystic ovary syndrome is known to be associated with insulin resistance in both lean and obese individuals. Insulin resistance in fact is felt to be a key feature in the reproductive and metabolic dysfunction of PCOS. There are numerous studies reporting the benefits of insulin sensitizing therapy, specifically metformin and thiazolidinediones, on the features of PCOS and emerging evidence on the impact of these agents on the risk and management of obesity. Weight loss and maintenance of weight reduction has been seen in women and adolescents treated with metformin therapy. Most studies indicate a synergy of metformin with lifestyle therapy in the general population but there are limited data in PCOS.
\end{abstract}

Keywords Obesity $\cdot$ Polycystic ovary syndrome $\cdot$ Weight reduction - Cardiovascular risk - Adolescents - Adolescent obesity $\cdot$ Metformin $\cdot$ Thiazolidinediones $\cdot$ Fertility risk Pregnancy complications $\cdot$ Insulin resistance $\cdot$ Type 2 diabetes $\cdot$ Insulin sensitizing therapy

K. M. Hoeger $(\bowtie)$

Department of Obstetrics and Gynecology, Division of Reproductive Endocrinology, University of Rochester Medical Center, 601 Elmwood Ave. Box 668,

Rochester, NY 14642, USA

e-mail: Kathy_hoeger@urmc.rochester.edu

\section{Introduction}

The association of obesity with PCOS has been noted since the first description of PCOS in the 1930s [1]. Despite this finding the diagnostic features of PCOS do not include obesity, but rather the diagnostic criteria include clinical or biochemical hyperandrogenism, oligoovulation and presence of polycystic ovaries on ultrasound [2]. There are serious sequelae to PCOS including reproductive dysfunction such as infertility and pregnancy complications, metabolic dysfunction including increased risk of type 2 diabetes and risk factors for cardiovascular disease. As such PCOS represents a major burden on healthcare irrespective of obesity. Obesity however is known to contribute both to the sequelae and the severity of these sequelae.

It is estimated that $40-60 \%$ of women with PCOS are obese although there is widespread variability in the degree of obesity based on geography. Studies range in prevalence from $20 \%$ in Spain to $69 \%$ in the United States [3, 4]. To some degree there is a correlation with the overall prevalence of obesity in the population, with the greatest prevalence in the general population of the United States at more than $30 \%$ [5]. The relative risk of obesity in PCOS is overall increased, with a meta-analysis suggesting a RR of 2.77 (1.80, 4.10). This is variable by race, with Caucasian women demonstrating a RR of 10.79 and Asian women a RR of 2.31 [6]. Obesity worsens the overall clinical picture of PCOS both reproductively and metabolically. Treatment of PCOS therefore must address the obesity as a primary intervention modality in order to adequately address the consequences of PCOS.

Insulin resistance is a major feature of PCOS and is particularly prevalent in those women who are obese with 70-80\% demonstrating insulin resistance and compensatory hyperinsulinemia [7]. Hyperinsulinemia is an important finding associated with the features of PCOS and addressing 
the hyperinsulinemia can be critical to the management of the disorder. Currently available insulin sensitizers that have been studied in PCOS include metformin and the thiazolidinedione therapies. With respect to the management of obesity in PCOS, there are studies supporting the use of insulin sensitizer. The majority of available evidence supporting insulin sensitizer use for obesity involves the use of metformin therapy, which will be the insulin sensitizer primarily reviewed in this article. The cornerstone of treatment of obesity in PCOS remains lifestyle therapy with dietary and behavioral changes. This has been previously reviewed [8]. However lifestyle therapy has notoriously low success rates over the long-term. While insulin sensitizing drugs are not considered weight loss agents, there is emerging evidence that they may play a role in the long-term management of weight reduction in both general obese populations and PCOS which will be reviewed.

\section{Impact of Obesity on Manifestations of PCOS}

There is evidence that obesity, particularly abdominal obesity, worsens both the clinical and endocrine features of PCOS [9]. The prevalence of obesity in PCOS is at least $50 \%$ in most studies. The phenotype of PCOS is strongly influenced by the presence of insulin resistance which is present in the majority of women diagnosed with PCOS by $\mathrm{NIH}$ criteria although this may be less so in those diagnosed by Rotterdam criteria [10]. Obesity influences the degree of insulin resistance, with obese women with PCOS demonstrating significantly more insulin resistance than their normal weight counterparts $[11,12]$. The converse of this may also be true with those demonstrating more severe insulin resistance also demonstrating a higher propensity to weight gain [13]. It is likely that obesity influences the clinical presentation of PCOS mediated in part through increased insulin resistance, worsening the clinical consequences. As such it is important to consider the impact of obesity on the features of PCOS when treating the condition.

\section{Impact of Obesity on Reproductive Features in PCOS}

The impact of obesity on both the reproductive and metabolic features of PCOS has been previously reviewed [14]. There is a clear impact of excess adiposity on the reproductive manifestations of PCOS. Very irregular menses, often associated with menorrhagia, is a complication of PCOS and likely represents oligo-ovulation, with lack of regular progestin withdrawal. Women with PCOS who demonstrate "regular" menstrual cycles, however may also be oligo-ovulatory despite the regular menses [15]. Obesity increases the frequency of anovulatory cycles in these women [16]. There is also an association of persistent anovulation, and continuous estrogen exposure, with endometrial hyperplasia due to lack of progestational changes to the endometrium. This may be associated with an increased risk of endometrial cancer [17]. It is unclear if the degree of menstrual irregularity is influenced by the degree of insulin resistance, but it appears to be influenced by obesity.

Both ovulatory and anovulatory infertility is increased in obesity. The majority of the impact on infertility in PCOS is likely mediated through anovulation however. A large epidemiologic study, the Nurses' Health Study, reported that the relative risk of infertility was significantly increased (1.3 fold higher) with BMI $>24-32 \mathrm{~kg} / \mathrm{m}^{2}$ and 2.7 fold higher with BMI $>32[18,19]$. Treatments for infertility in the setting of ovulatory dysfunction frequently involve use of ovulation induction agents. There is good evidence that dosing requirements are increased in women with obesity [20]. Using a calculated model to determine those women with PCOS who were most likely to fail to respond to clomiphene citrate in a large population from the Netherlands, BMI in addition to testosterone concentrations, was the lead predictor of failure to respond [21].

Treatment protocols for infertility may include the use of gonadotropin therapy if clomiphene is unsuccessful in inducing ovulation [22]. Gonadotropin dosing requirement is increased in obesity and there is an increased cancellation rate due to failure to produce adequate follicular development in many women with obesity undergoing gonadotropin stimulation [23]. There is some evidence that treatment with IVF may also be less successful in obese women with PCOS. There is evidence of increased requirements for gonadotropins, lower oocyte yield and fertilization rates, as well as high cycle cancellation rates [24]. Whether these findings are related to the presence of increased insulin resistance is unclear, but these data taken in total indicate obesity clearly influences both the need for and the success of fertility treatments in PCOS.

A defining characteristic of PCOS is excess androgen production associated with symptoms such as hirsutism, which may be exacerbated in the presence of obesity [25]. The degree of insulin resistance is correlated with the degree of hyperandrogenism. Obese women with PCOS have more severe insulin resistance, compared to lean PCOS women [11]. The increased insulin resistance may drive abnormal sex steroid production. Sex hormone binding globulin (SHBG) is the major binding globulin for testosterone and suppression of SHBG that is seen in insulin resistant states is associated with increased concentration of circulating free testosterone resulting in a worse clinical picture [26]. Obese women with PCOS have higher free testosterone concentration compare to normal weight women with PCOS. Hirsutism may also be worse, with obesity acting synergistically with the intrinsic endocrine disturbance in PCOS [16].

Obesity's influence is seen early on in reproductive development. Obese pre-pubertal girls demonstrate higher serum androgen concentrations when compared to normal weight pre-pubertal controls [27]. Puberty enhances insulin 
resistance in the natural state with a resultant normal increase in serum androgens. In the setting of obesity however, puberty is associated with a two-fold increase in serum testosterone compared with that seen in lean pubertal girls [28, 29].

It is likely that a majority of the detrimental reproductive features of obesity in PCOS are mediated in part by insulin resistance that is exacerbated in obese individuals. Both androgen excess as well as ovulatory dysfunction are worse in the setting of obesity. A summary of the impact of obesity in PCOS is shown in Table 1.

\section{Impact of Obesity on Pregnancy Complications in PCOS}

Women with PCOS, particularly those who are obese are at significant risk for complications during pregnancy. Pregnancy itself is an insulin resistant state which may be exaggerated in PCOS. A meta-analysis of pregnancy complications in PCOS found that women with PCOS had a significantly higher rate of gestational diabetes mellitus, pregnancy induced hypertension, preeclampsia and preterm birth [30]. Subsequently an updated meta-analysis, was published in 2011 that confirmed these increased risks with a stronger association with PCOS and hypertensive disorders [31].

A recent cross-sectional study of 257 women with PCOS in Norway looked at the incidence of pregnancy complications in the first trimester in a large cohort of women with PCOS [32•]. Women were classified as PCOS by either NIH [33] or Rotterdam criteria. BMI was higher in those diagnosed by NIH criteria which may represent a more severe phenotype and women diagnosed by NIH criteria were more metabolically and endocrinologically abnormal in this study. BMI was an independent predictor for elevated blood pressure in the first trimester and was also correlated to both fasting and $2 \mathrm{~h}$ glucose. There was a high prevalence of gestational diabetes by early screen in the first trimester in these PCOS pregnancies, none of whom had known diabetes before conceiving and normal fasting glucose levels.

Table 1 Impact of obesity on PCOS

\author{
Endocrine features \\ 个estrogen \\ $\uparrow$ androgens \\ $\downarrow$ SHBG \\ Reproductive features \\ $\downarrow$ clinical pregnancy rates \\ $\uparrow$ miscarriage \\ $\downarrow$ decreased live birth rate \\ $\uparrow$ pregnancy complications \\ Metabolic features \\ $\uparrow$ insulin resistance \\ $\uparrow$ diabetes risk \\ $\uparrow$ inflammatory markers
}

Cardiovascular Risk in PCOS and the Impact of Obesity

Whether cardiovascular disease is increased in PCOS is a matter of debate [34]. However there is little disagreement that the individual risk factors of cardiovascular disease may be increased in PCOS, particularly in the setting of obesity. The relative independent contributions of obesity and PCOS to cardiovascular risk are still unclear, but likely mediated via increased insulin resistance. Subclinical measures of cardiovascular risk have been studied in PCOS. A recent systematic review summarized the current state of cardiovascular risk in PCOS [35]. Women with PCOS have elevated triglycerides, low HDL-cholesterol and elevated LDLcholesterol. Increased carotid intima medial thickness, a known predictor of myocardial infarction and stroke, is increased in women with PCOS which may be mediated in part due to obesity [36]. Coronary artery calcification (CAC) is also a known risk factor for cardiovascular disease. CAC is increased in women with PCOS and exacerbated by obesity [37].

Metabolic syndrome is a constellation of cardiovascular risk factors linked via insulin resistance that is associated with increased cardiovascular risk [38]. In the general population, the presence of metabolic syndrome, in the absence of type 2 diabetes, confers a two-fold greater risk of cardiovascular events and five-fold if there is concurrent diabetes $[39,40]$. The increased risk of cardiovascular disease seen in metabolic syndrome may be linked to inflammation associated with increased insulin resistance, as evidenced by markers such as increased hs-CRP [41].

Risk of metabolic syndrome may be increased in women with PCOS compared to healthy controls, with reported incidences ranging from $43 \%$ to $47 \%$ [42-44]. Inflammatory markers are linked to increased risk of metabolic syndrome in PCOS, factors that are likely linked to both obesity and insulin resistance [45].

Increased prevalence of metabolic syndrome has also been demonstrated in some studies in adolescents diagnosed with PCOS. A study of adolescent women with PCOS demonstrated a $37 \%$ incidence of metabolic syndrome, which was higher that than reported in a national sample of obese adolescents [46]. Overall diagnosis of PCOS was associated with a four-fold greater risk of metabolic syndrome. However metabolic syndrome may not be independent of obesity in PCOS as other studies did not demonstrate differences when compared to obese non-PCOS adolescents. When compared to a control group matched closely for weight and BMI, PCOS did not seem to infer additional risk independent of obesity $[47,48]$. Overall while there is a paucity of data linking PCOS and cardiovascular mortality, the risk factors for cardiovascular disease appear to be increased, beginning in early adolescence in PCOS, and increased in the setting of obesity. 


\section{Insulin Resistance in PCOS}

Insulin resistance was first identified in women with PCOS in the 1980s [49]. It is felt to be a common but not universal phenotype in PCOS. Hepatic insulin resistance is seen only in women with PCOS who are obese, suggesting there is a synergy between PCOS and obesity with respect to insulin resistance [50]. Pancreatic $\beta$-cell dysfunction is seen in those individuals with other type 2 diabetes risk factors and predisposes to development of diabetes [51].

There is some evidence that women with androgen excess may have increased upper body fat (android obesity) which may predispose to increased rates of hyperinsulinemia and insulin resistance [52]. More recent studies however have challenged the presumption that women with PCOS have increased visceral adiposity [53]. However, there is evidence that adipose tissue in obese women with PCOS may behave differently from women without PCOS. This includes an increase in rates of lipolysis and increased release of free fatty acids, which may predispose to insulin resistance [54].

There is considerable evidence that the hyperinsulinemia, noted in a majority of women with PCOS, contributes to the reproductive and metabolic dysfunction of the condition, but whether insulin resistance is a primary defect in PCOS is not clear. It is possible that the insulin resistance seen in PCOS is a modifying factor rather than causative, given the wide variability in presentations seen. Nonetheless insulin resistance is a potential powerful target for medical management of the syndrome, particularly in the setting of obesity.

\section{Insulin Sensitizing Agents and the Impact on Obesity}

While the cornerstone of treatment of obesity should be founded in lifestyle changes in diet and exercise, there are low success rates in achieving and maintaining weight loss. Therefore drug therapy may offer additional benefits to achieving weight reduction [55]. However the traditional drugs used for weight reduction may not be suitable for women attempting conception as they have not been tested in early pregnancy. Insulin sensitizing agents are not traditional weight loss agents or considered antiobesity drugs. There is some evidence, however, that metformin therapy may contribute to weight reduction. Weight reduction in the metformin arm of the diabetes prevention trial was $-2.08 \%$ compared to $-0.2 \%$ in the placebo arm [56].

Obesity predisposes individuals to type 2 diabetes mellitus. Fasting hyperinsulinemia in insulin resistant states predates the onset of type 2 diabetes by several years and post-prandial rises in glycemia can be present several years before detection of formal diagnosis of diabetes as evidenced by abnormal $2 \mathrm{~h}$ glucose tolerance testing prior to development of fasting hyperglycemia. Women with PCOS and obesity have an increased rate of conversion to type 2 diabetes in a recent [57] long term study with an age-standardized prevalence of $39.3 \%$ at the end of the study. Therefore management of insulin resistance before the onset of diabetes is imperative and has been shown to delay the onset of diabetes [56, 58].

Maintenance of weight loss once achieved is also challenging. In a 7-8 year follow up to the diabetes prevention program where metformin was used open label, the initial weight loss was maintained and at the end of a 10 year assessment, weight reduction was $2 \%$ in the metformin group. This was directly related to metformin continuation [59••]. Additionally serum liver function tests are often elevated in obesity associated with nonalcoholic fatty liver disease (NAFLD). Subjects in the DPP randomized to the metformin arm demonstrated reduction in ALT over the first 2 years of the study but this was directly related to weight loss associated with metformin use [60].

\section{Impact in PCOS}

An initial systemic review of the effectiveness of metformin in PCOS did not suggest an impact on weight loss [61]. This meta-analysis however was focused on the use of metformin in ovulation induction in PCOS and did find improvement in ovulatory parameters and menstrual cycles with metformin use. Subsequent large randomized trials however have demonstrated modest weight reduction in the metformin arms of treatment. In the Pregnancy in Polycystic Ovary Syndrome Study (PPCOS) which included randomization to clomiphene, clomiphene plus metformin and metformin alone, the metformin arm demonstrated a significant mean reduction in BMI of -0.6 with the combination group demonstrating a decrease in BMI of -0.5 . This is compared to the clomiphene arm which demonstrated a significant net increase in BMI of +0.2 . Despite the weight reduction, metformin was not better than placebo in inducing pregnancy [62].

Weight loss has been seen in a number of studies of insulin sensitizers in women with obesity. A systematic review of use of insulin sensitizers in women of reproductive age included 14 randomized placebo controlled trials and included trials with metformin as well as thiazolidinediones. Comparing all randomized placebo controlled metformin studies, an overall BMI reduction of -0.68 was seen $(-1.13,-0.24)$ favoring metformin use. When analyzed by dose of metformin used in the trials, this difference was only significant for the high dose $(>1,500 \mathrm{mg})$ therapy. Studies of short duration $(<8$ weeks) also did not demonstrate a benefit [63].

A recent randomized, placebo-controlled trial of metformin in women attempting pregnancy in Finland compared the pregnancy rates after 3 months of treatment. The investigators gave $2,000 \mathrm{mg}$ per day to obese women and $1,500 \mathrm{mg}$ to nonobese women. The pregnancy rate was significantly higher in the obese group treated with metformin compared to placebo, although pregnancy rate was not significantly improved in the 
non-obese group. The metformin subjects demonstrated a $1.3 \mathrm{~kg}$ weight loss over the 3 months study which was not seen in the placebo group. It is not clear if the improvement in pregnancy rates was related in part to the weight reduction [64-]. Overall this suggests that use of metformin in reproductive aged women with obesity and PCOS leads to a significant but modest weight reduction that is influenced by both dose and duration of treatment. This weight reduction may improve pregnancy rates. Weight loss alone in PCOS produces variable phenotypic changes in PCOS but more than $1 / 3$ will have resolution of PCOS phenotype [65].

There are limited trials utilizing thiazolidinedione therapy alone and in comparison to metformin. Overall there was no evidence of a differential in weight loss seen but these comparative trials were small in size with limited power [66-68]. Thiazolidinedione therapy has been shown in non-PCOS populations to contribute to weight gain $[69,70]$.

What about the impact of insulin sensitizing agents on metabolic complications associated with obesity in women with PCOS? There are few data in women with PCOS alone, but in those at risk for diabetes, metformin was demonstrated to decrease fasting glucose, triglycerides and LDL significantly. Additionally there is improvement in fasting insulin and reduced insulin resistance as well as reduction in risk of type 2 diabetes mellitus. In a meta-analysis of placebo controlled studies of metformin, with a subanalysis of women with PCOS, women with PCOS had a weight reduction on metformin of $-5.3 \%$ compared to placebo. These women had a BMI of 33.9 at baseline [71]. Overall metformin improved triglycerides $-5.3 \%$ compared to placebo and LDL was reduced $5.6 \%$.

With respect to impact of metformin on pregnancy complications in women with PCOS, there is little data to support a benefit of metformin use. A randomized, doubleblind, multicenter trial in Norway there was no improvement of risk of pre-eclampsia, gestational diabetes in women randomized to metformin [72••]. Metformin did reduce the amount of weight gained during pregnancy but this did not alter birth weight. The findings were not different in women with BMI $>30 \mathrm{~kg} / \mathrm{m}^{2}$ as demonstrated in a subgroup analysis, suggesting that obesity alone is not an indication for metformin use in pregnancy in women with PCOS.

\section{Insulin Sensitizing Agents and the Impact on Adolescents with Obesity and PCOS}

PCOS is known to present in the perimenarchal time period. Hyperandrogenism is known to be exacerbated in pubertal girls who are obese [27]. Obese girls with hyperandrogenism are very likely to be at risk for development of PCOS, although long-term studies of this have not been reported. Adolescence is a time of marked insulin resistance that is exaggerated in obese adolescents. It is likely that this group could be a target for management of insulin resistance with potential for improved long-term health.

Metformin has been studied in obese adolescents with and without PCOS. Metformin for 3 months in obese hyperandrogenic adolescents reduced androgenic response to ACTH [73]. Metformin, in combination with oral contraceptives and lifestyle modification, in another small trial in adolescents with PCOS, did not improve weight reduction compared to placebo but did appear to result in decreased waist circumference [74].

Metformin has been studied more extensively in adolescents with general obesity. In a 2009 systematic review of five randomized controlled trials of metformin in obese children and adolescents suggested that metformin appeared to be efficacious for weight reduction. Overall metformin treatment of at least 6 months duration reduced BMI by $1.42 \mathrm{~kg} / \mathrm{m}^{2}$ [75]. In a subsequent trial using metformin or placebo, in combination with lifestyle modification, was initiated after initial unsuccessful lifestyle intervention for 6 months. There was no significant reduction in BMI in the metformin group compared to placebo although insulin resistance improved. The subjects received $1,000 \mathrm{mg}$ per day of metformin in this study [76 $]$.

In a 12 week study of $1,500 \mathrm{mg}$ of metformin or placebo or comprehensive lifestyle therapy in 203 overweight or obese young women, metformin was not effective in reducing body weight whereas lifestyle modification demonstrated a mean of $-4.2 \mathrm{~kg}$ in weight reduction [77•].

The impact of metformin may be related to timing of the start as well as the dose. In a 6-month study of children aged 612 with severe obesity, children randomized to $2,000 \mathrm{mg}$ of metformin daily reduced weight by $-3.38 \mathrm{~kg}$ during the placebo controlled phase of the study. The study was then extended in an open label protocol. No additional weight reduction was seen in the metformin group, but a significant reduction in BMI was noted in those previously treated with placebo who were then given metformin [78•]. In a longer 48 week

Table 2 Clinical Impact of Insulin Sensitizers on obese PCOS

\begin{tabular}{ll}
\hline Feature & Impact of insulin sensitizer \\
\hline Weight loss & $\begin{array}{c}\text { Consistent weight reduction of } \\
2-3 \% \text { with metformin, dose } \\
\text { and time dependent }\end{array}$ \\
$\begin{array}{l}\text { Menstrual frequency } \\
\text { Improved in majority of trials }\end{array}$ \\
$\begin{array}{l}\text { Improved in majority of trials } \\
\text { Miscarriage }\end{array}$ & Variable in large trials \\
Pregnancy complications & No impact in majority of trials \\
Glucose tolerance & No impact \\
Cardiovascular risk markers & Improved \\
Mortality & Some evidence of improved lipids, \\
\end{tabular}


randomized placebo controlled trial of obese adolescents with obesity, an escalating dose of metformin up to $2,000 \mathrm{mg}$ was used. Metformin use was associated with a significant impact on body weight over a period of 52 weeks with a reduction in BMI of -0.9 . The placebo group did not demonstrate a reduction in BMI despite lifestyle advice. The BMI reduction persisted in the metformin group for 12-24 weeks after discontinuation of study drug after which body weight increased toward that in the control group [79]. A summary of the impact of insulin sensitizers in PCOS is shown in Table 2.

\section{Conclusions}

PCOS is associated with insulin resistance in the majority of cases with or without obesity but insulin resistance is increased in obese individuals. As obesity is present in a majority of individuals with PCOS, with a significant risk of developing type 2 diabetes mellitus, insulin resistance is a reasonable therapy target in PCOS treatment paradigms. Studied insulin sensitizing agents in PCOS include metformin and thiazolidinediones. As thiazolidinedione therapy has been associated with weight gain as well as other potential cardiovascular risks, the majority of trials of insulin sensitizing therapy in PCOS have focused on the use of metformin. While lifestyle modification is the cornerstone of therapy for obesity related concerns in PCOS, there is overall low compliance with therapy and difficulty with sustaining lifestyle changes over time. There is reasonable evidence that metformin is associated with a sustained and reproducible weight loss in obese individuals. Data specifically in PCOS are limited but are consistent with this finding. Data from the general population are encouraging.

The success of metformin with respect to weight loss may be related to both duration of treatment and the dose, with doses $<1,500 \mathrm{mg}$ not demonstrating a significant weight impact. Studies suggest longer duration may be of more benefit but that maximal benefit may be seen by 6 months into therapy. The age of metformin start may also be relevant to its success. Data in children and adolescents with obesity are remarkably consistent for weight reduction and this may be a significant tool in the early management of PCOS in adolescents. There are limited data on metformin's impact on metabolic parameters in PCOS but overall there is significant impact on conversion to type 2 diabetes in the general population as well as improvement in triglycerides and LDL in obese individuals.

Use of any drugs in reproductive aged women has to consider the impact on pregnancy. Although metformin has not been demonstrated to improve pregnancy outcomes in obese women with PCOS, there is a generally good safety profile compared to other insulin sensitizing agents. Overall the role for metformin in obesity in PCOS is evolving but data suggest favorable impact on both weight and metabolic indices. Early treatment in adolescence is intriguing and deserving of further large scale trials in adolescents at risk for PCOS.

Acknowledgment K.M. Hoeger has submitted a grant on PCOS to the NIH, with pending funding.

Disclosure No potential conflicts of interest relevant to this article were reported.

\section{References}

Papers of particular interest, published recently, have been highlighted as:

- Of importance

-. Of major importance

1. Stein IF ML. Amenorrhea associated with bilateral polycystic ovaries. Am J Obstet Gynecol. 1935;29:181-191.

2. The ESHRE/ASRM-Sponsored PCOS Consensus Workshop Group. Revised 2003 consensus on diagnostic criteria and longterm health risks related to polycystic ovary syndrome. Fertil Steril. 2004;81:19-25.

3. Asuncion M, Calvo RM, San Millan JL, Sancho J, Avila S, Escobar-Morreale HF. A prospective study of the prevalence of the polycystic ovary syndrome in unselected Caucasian women from Spain. J Clin Endocrinol Metab. 2000;85:2434-8.

4. Carmina E. Genetic and environmental aspect of polycystic ovary syndrome. J Endocrinol Invest. 2003;26:1151-9.

5. Freedman DS. Obesity - United States, 1988-2008. MMWR Surveill Summ. 2011;60(Suppl):73-7.

6. Lim SS, Davies MJ, Norman RJ, Moran LJ. Overweight, obesity and central obesity in women with polycystic ovary syndrome: a systematic review and meta-analysis. Hum Reprod Update. 2012; Jul 4. [Epub ahead of print].

7. DeUgarte CM, Bartolucci AA, Azziz R. Prevalence of insulin resistance in the polycystic ovary syndrome using the homeostasis model assessment. Fertil Steril. 2005;83:1454-60.

8. Moran LJ, Pasquali R, Teede HJ, Hoeger KM, Norman RJ. Treatment of obesity in polycystic ovary syndrome: a position statement of the Androgen Excess and Polycystic Ovary Syndrome Society. Fertil Steril. 2009;92:1966-82.

9. Pasquali R, Casimirri F, Venturoli S, et al. Body fat distribution has weight-independent effects on clinical, hormonal, and metabolic features of women with polycystic ovary syndrome. Metabolism. 1994;43:706-13.

10. Kauffman RP, Baker TE, Baker VM, DiMarino P, Castracane VD. Endocrine and metabolic differences among phenotypic expressions of polycystic ovary syndrome according to the 2003 Rotterdam consensus criteria. Am J Obstet Gynecol. 2008;198:670 e1-7. discussion 670 e 7-10.

11. Dunaif A, Segal KR, Futterweit W, Dobrjansky A. Profound peripheral insulin resistance, independent of obesity, in polycystic ovary syndrome. Diabetes. 1998;38:1165-74.

12. Morales AJ, Laughlin GA, Butzow T, Maheshwari H, Baumann G, Yen SS. Insulin, somatotropic, and luteinizing hormone axes in lean and obese women with polycystic ovary syndrome: common and distinct features. J Clin Endocrinol Metab. 1996;81:2854-64.

13. Robinson S, Chan SP, Spacey S, Anyaoku V, Johnston DG, Franks $\mathrm{S}$. Postprandial thermogenesis is reduced in polycystic ovary 
syndrome and is associated with increased insulin resistance. Clin Endocrinol (Oxf). 1992;36:537-43.

14. Hoeger KM. Obesity and lifestyle management in polycystic ovary syndrome. Clin Obstet Gynecol. 2007;50:277-94.

15. Carmina E, Chu MC, Longo RA, Rini GB, Lobo RA. Phenotypic variation in hyperandrogenic women influences the findings of abnormal metabolic and cardiovascular risk parameters. J Clin Endocrinol Metab. 2005;90:2545-9.

16. Kiddy DS, Sharp PS, White DM, et al. Differences in clinical and endocrine features between obese and non-obese subjects with polycystic ovary syndrome: an analysis of 263 consecutive cases. Clin Endocrinol (Oxf). 1990;32:213-20.

17. Webb PM. Commentary: weight gain, weight loss, and endometrial cancer. Int J Epidemiol. 2006;35:166-8.

18. Rich-Edwards JW, Spiegelman D, Garland M, et al. Physical activity, body mass index, and ovulatory disorder infertility. Epidemiology. 2002;13:184-90.

19. Rich-Edwards JW, Goldman MB, Willett WC, et al. Adolescent body mass index and infertility caused by ovulatory disorder. Am J Obstet Gynecol. 1994;171:171-7.

20. Dickey RP, Taylor SN, Curole DN, Rye PH, Lu PY, Pyrzak R. Relationship of clomiphene dose and patient weight to successful treatment. Hum Reprod. 1997;12:449-53.

21. Imani B, Eijkemans M, Te Velde ER, Habbeman JDF, Fauser BCJM. Predictors of patients remaining anovulatory during colimphene citrate induction of ovulation in normogonadotropic oligomenorrheic infertility. J Clin Endocrinol Metab. 1998;83:2361-5.

22. The ESHRE/ASRM-Sponsored PCOS Consensus Workshop Group. Consensus on infertility treatment related to polycystic ovary syndrome. Fertil Steril. 2008;89:505-22.

23. Hamilton-Fairley D, Kiddy D, Watson H, Paterson C, Franks S. Association of moderate obesity with a poor pregnancy outcome in women with polycystic ovary syndrome treated with low dose gonadotrophin. Br J Obstet Gynaecol. 1992;99:128-31.

24. Fedorcsak P, Dale PO, Storeng R, et al. Impact of overweight and underweight on assisted reproduction treatment. Hum Reprod. 2004;19:2523-8.

25. Dunaif A, Mandeli J, Fluhr H, Dobrjansky A. The impact of obesity and chronic hyperinsulinemia on gonadotropin release and gonadal steroid secretion in the polycystic ovary syndrome. J Clin Endocrinol Metab. 1988;66:131-9.

26. Lukanova A, Lundin E, Zeleniuch-Jacquotte A, et al. Body mass index, circulating levels of sex-steroid hormones, IGF-I and IGFbinding protein-3: a cross-sectional study in healthy women. Eur J Endocrinol. 2004;150:161-71.

27. McCartney CR, Prendergast KA, Chhabra S, et al. The association of obesity and hyperandrogenemia during the pubertal transition in girls: obesity as a potential factor in the genesis of postpubertal hyperandrogenism. J Clin Endocrinol Metab. 2006;91:1714-22.

28. Reinehr T, de Sousa G, Roth CL, Andler W. Androgens before and after weight loss in obese children. J Clin Endocrinol Metab. 2005;90:5588-95.

29. McCartney CR, Blank SK, Prendergast KA, et al. Obesity and sex steroid changes across puberty: evidence for marked hyperandrogenemia in pre- and early pubertal obese girls. J Clin Endocrinol Metab. 2007;92:430-6.

30. Boomsma CM, Eijkemans MJ, Hughes EG, Visser GH, Fauser BC, Macklon NS. A meta-analysis of pregnancy outcomes in women with polycystic ovary syndrome. Hum Reprod Update. 2006;12:673-83.

31. Kjerulff LE, Sanchez-Ramos L, Duffy D. Pregnancy outcomes in women with polycystic ovary syndrome: a metaanalysis. Am J Obstet Gynecol. 2011;204:558 e1-6.

32. - Vanky E, Stridsklev S, Skogoy K, et al. PCOS-what matters in early pregnancy?-data from a cross-sectional, multicenter study. Acta Obstet Gynecol Scand. 2011;90:398-404. This study demonstrates a signficant relationship of both hypertension and diabetes risk in PCOS associated with obesity.

33. Zawadzki JK, Dunaif A. Diagnositic criteria for polycystic ovary syndrome: towards a rational approach. In: Dunaif A, Givens J, Haseltine FP, Merriam GR, editors. Polycystic ovary syndrome. Blackwell: Oxford; 1992. p. 377-84.

34. Lo JC, Feigenbaum SL, Yang J, Pressman AR, Selby JV, Go AS. Epidemiology and adverse cardiovascular risk profile of diagnosed polycystic ovary syndrome. J Clin Endocrinol Metab. 2006;91:1357-63.

35. Wild RA, Carmina E, Diamanti-Kandarakis E, et al. Assessment of cardiovascular risk and prevention of cardiovascular disease in women with the polycystic ovary syndrome: a consensus statement by the Androgen Excess and Polycystic Ovary Syndrome (AEPCOS) Society. J Clin Endocrinol Metab. 2010;95:2038-49.

36. Talbott EO, Zborowski JV, Boudreaux MY, McHugh-Pemu KP, Sutton-Tyrrell K, Guzick DS. The relationship between C-reactive protein and carotid intima-media wall thickness in middle-aged women with polycystic ovary syndrome. J Clin Endocrinol Metab. 2004;89:6061-7.

37. Shroff R, Kerchner A, Maifeld M, Van Beek EJ, Jagasia D, Dokras A. Young obese women with polycystic ovary syndrome have evidence of early coronary atherosclerosis. J Clin Endocrinol Metab. 2007;92:4609-14.

38. Royer M, Castelo-Branco C, Blumel JE, et al. The US National Cholesterol Education Programme Adult Treatment Panel III (NCEP ATP III): prevalence of the metabolic syndrome in postmenopausal Latin American women. Climacteric. 2007;10:164-70.

39. Malik S, Wong ND, Franklin SS, et al. Impact of the metabolic syndrome on mortality from coronary heart disease, cardiovascular disease, and all causes in United States adults. Circulation. 2004;110:1245-50.

40. McNeill AM, Rosamond WD, Girman CJ, et al. The metabolic syndrome and 11-year risk of incident cardiovascular disease in the atherosclerosis risk in communities study. Diabetes Care. 2005;28:385-90.

41. Bo S, Rosato R, Ciccone G, et al. What predicts the occurrence of the metabolic syndrome in a population-based cohort of adult healthy subjects? Diabetes Metab Res Rev. 2009;25:76-82.

42. Ehrmann DA, Liljenquist DR, Kasza K, Azziz R, Legro RS, Ghazzi MN. Prevalence and Predictors of the Metabolic Syndrome in Women with Polycystic Ovary Syndrome (PCOS). J Clin Endocrinol Metab. 2006;91(1):48-53.

43. Apridonidze T, Essah PA, Iuorno MJ, Nestler JE. Prevalence and characteristics of the metabolic syndrome in women with polycystic ovary syndrome. Obstet Gynecol Surv. 2005;60:589-91.

44. Teede HJ, Hutchison S, Zoungas S, Meyer C. Insulin resistance, the metabolic syndrome, diabetes, and cardiovascular disease risk in women with PCOS. Endocrine. 2006;30:45-53.

45. Tosi F, Dorizzi R, Castello R, et al. Body fat and insulin resistance independently predict increased serum C-reactive protein in hyperandrogenic women with polycystic ovary syndrome. Eur J Endocrinol. 2009;161:737-45.

46. Coviello AD, Legro RS, Dunaif A. Adolescent girls with polycystic ovary syndrome have an increased risk of the metabolic syndrome associated with increasing androgen levels independent of obesity and insulin resistance. J Clin Endocrinol Metab. 2006;91:492-7.

47. Rossi B, Sukalich S, Droz J, et al. Prevalence of metabolic syndrome and related characteristics in obese adolescents with and without polycystic ovary syndrome. J Clin Endocrinol Metab. 2008;93:4780-6.

48. Vrbikova J, Zamrazilova H, Sedlackova B, Snajderova M. Metabolic syndrome in adolescents with polycystic ovary syndrome. Gynecol Endocrinol. 2011;27:820-2.

49. Dunaif A, Graf M, Mandeli J, Laumas V, Dobrjansky A. Characterization of groups of hyperandrogenic women with acanthosis 
nigricans, impaired glucose tolerance, and/or hyperinsulinemia. J Clin Endocrinol Metab. 1987;65:499-507.

50. Sam S, Dunaif A. Polycystic ovary syndrome: syndrome XX? Trends Endocrinol Metab. 2003;14:365-70.

51. Bergman RN, Kim SP, Hsu IR, et al. Abdominal obesity: role in the pathophysiology of metabolic disease and cardiovascular risk. Am J Med. 2007;120:S3-8. discussion S29-32.

52. Kirschner MA, Samojlik E, Drejka M, Szmal E, Schneider G, Ertel $\mathrm{N}$. Androgen-estrogen metabolism in women with upper body versus lower body obesity. J Clin Endocrinol Metab. 1990;70:473-9.

53. Barber TM, Golding SJ, Alvey C, et al. Global adiposity rather than abnormal regional fat distribution characterizes women with polycystic ovary syndrome. J Clin Endocrinol Metab. 2008;93:999-1004.

54. Ek I, Arner P, Ryden M, et al. A unique defect in the regulation of visceral fat cell lipolysis in the polycystic ovary syndrome as an early link to insulin resistance. Diabetes. 2002;51:484-92.

55. Wadden TA, Berkowitz RI, Womble LG, et al. Randomized trial of lifestyle modification and pharmacotherapy for obesity. N Engl J Med. 2005;353:2111-20.

56. Knowler WC, Barrett-Connor E, Fowler SE, et al. Reduction in the incidence of type 2 diabetes with lifestyle intervention or metformin. N Engl J Med. 2002;346:393-403.

57. Gambineri A, Patton L, Altieri P, et al. Polycystic ovary syndrome is a risk factor for type 2 diabetes: results from a long-term prospective study. Diabetes Diabetes. 2012 Jun 14. [Epub ahead of print]).

58. Tuomilehto J, Lindstrom J, Eriksson JG, et al. Prevention of type 2 diabetes mellitus by changes in lifestyle among subjects with impaired glucose tolerance. N Engl J Med. 2001;344:1343-50.

59. •- Diabetes Prevention Program Research Group. Long-term safety, tolerability, and weight loss associated with metformin in the Diabetes Prevention Program Outcomes Study. Diabetes Care. 2012;35:731-7. This is a long term follow up report to the diabetes prevention program and demonstrates both long term safety and efficacy of metformin as a meaningful strategy in diabetes prevention. The study encompasses 10 years of follow up in total.

60. Greenhill C. Metformin, weight loss and NAFLD. Nat Rev Endocrinol. 2010;6:296.

61. Lord JM, Flight IH, Norman RJ. Metformin in polycystic ovary syndrome: systematic review and meta-analysis. BMJ. 2003;327:951-3.

62. Legro RS, Barnhart HX, Schlaff WD, et al. Clomiphene, metfor$\mathrm{min}$, or both for infertility in the polycystic ovary syndrome. $\mathrm{N}$ Engl J Med. 2007;356:551-66.

63. Nieuwenhuis-Ruifrok AE, Kuchenbecker WK, Hoek A, Middleton $\mathrm{P}$, Norman RJ. Insulin sensitizing drugs for weight loss in women of reproductive age who are overweight or obese: systematic review and meta-analysis. Hum Reprod Update. 2009;15:57-68.

64. - Morin-Papunen L, Rantala AS, Unkila-Kallio L, et al. Metformin improves pregnancy and live-birth rates in women with polycystic ovary syndrome (PCOS): a multicenter, double-blind, placebocontrolled randomized trial. J Clin Endocrinol Metab. 2012;97:1492-500. This multicenter trial in Finland demonstrates the benefit of pretreatment metformin in obese women with PCOS attempting pregnancy.

65. Pasquali R, Gambineri A, Cavazza C, et al. Heterogeneity in the responsiveness to long-term lifestyle intervention and predictability in obese women with polycystic ovary syndrome. Eur J Endocrinol. 2011;164:53-60.

66. Kilicdag EB, Bagis T, Zeyneloglu HB, et al. Homocysteine levels in women with polycystic ovary syndrome treated with metformin versus rosiglitazone: a randomized study. Hum Reprod. 2005;20:894-9.
67. Mitkov M, Pehlivanov B, Terzieva D. Metformin versus rosiglitazone in the treatment of polycystic ovary syndrome. Eur J Obstet Gynecol Reprod Biol. 2006;126:93-8.

68. Ortega-Gonzalez C, Luna S, Hernandez L, et al. Responses of serum androgen and insulin resistance to metformin and pioglitazone in obese, insulin-resistant women with polycystic ovary syndrome. J Clin Endocrinol Metab. 2005;90:1360-5.

69. Kahn SE, Haffner SM, Heise MA, et al. Glycemic durability of rosiglitazone, metformin, or glyburide monotherapy. N Engl J Med. 2006;355:2427-43.

70. Balas B, Belfort R, Harrison SA, et al. Pioglitazone treatment increases whole body fat but not total body water in patients with non-alcoholic steatohepatitis. J Hepatol. 2007;47:565-70.

71. Salpeter SR, Buckley NS, Kahn JA, Salpeter EE. Meta-analysis: metformin treatment in persons at risk for diabetes mellitus. Am J Med. 2008;121:149-157 e2.

72. •• Vanky E, Stridsklev S, Heimstad R, et al. Metformin versus placebo from first trimester to delivery in polycystic ovary syndrome: a randomized, controlled multicenter study. J Clin Endocrinol Metab. 2010;95:E448-55. This is the first multicenter placebo controlled trial of metformin use in pregnancy. There was no demonstrated benefit on pregnancy complications in obese women with PCOS using metformin in pregnancy.

73. Arslanian SA, Lewy V, Danadian K, Saad R. Metformin therapy in obese adolescents with polycystic ovary syndrome and impaired glucose tolerance: amelioration of exaggerated adrenal response to adrenocorticotropin with reduction of insulinemia/insulin resistance. J Clin Endocrinol Metab. 2002;87:1555-9.

74. Hoeger K, Davidson K, Kochman L, Cherry T, Kopin L, Guzick DS. The impact of metformin, oral contraceptives, and lifestyle modification on polycystic ovary syndrome in obese adolescent women in two randomized, placebo-controlled clinical trials. J Clin Endocrinol Metab. 2008;93:4299-306.

75. Park MH, Kinra S, Ward KJ, White B, Viner RM. Metformin for obesity in children and adolescents: a systematic review. Diabetes Care. 2009;32:1743-5.

76. - Wiegand S, l'Allemand D, Hubel H, et al. Metformin and placebo therapy both improve weight management and fasting insulin in obese insulin-resistant adolescents: a prospective, placebocontrolled, randomized study. Eur J Endocrinol. 2010;163:58592. This study demonstrated improvement in metabolic parameters with lifestyle intervention in obese adolescents but did not demonstrate additional metformin benefit. However a low dose of metformin was used.

77. - Lim SS, Norman RJ, Clifton PM, Noakes M. The effect of comprehensive lifestyle intervention or metformin on obesity in young women. Nutr Metab Cardiovasc Dis. 2011;21:261-8. In this trial effective lifestyle intervention was demonstrated in over 200 obese young women. There was no additional benefit seen to metformin use.

78. - Yanovski JA, Krakoff J, Salaita CG, et al. Effects of metformin on body weight and body composition in obese insulin-resistant children: a randomized clinical trial. Diabetes. 2011;60:477-85. In this randomized trial of metformin or placebo in obese children, metformin reduced body weight and improved body composition.

79. Wilson DM, Abrams SH, Aye T, et al. Metformin extended release treatment of adolescent obesity: a 48-week randomized, doubleblind, placebo-controlled trial with 48-week follow-up. Arch Pediatr Adolesc Med. 2010;164:116-23. 